\title{
THE EFFECT OF NEURAL MOBILISATION ON BACK PAIN IN PATIENTS WITH ADULT SCOLIOSIS
}

\author{
Evgeniya Dimitrova
}

National Sports Academy "Vassil Levski", Sofia, Bulgaria

\begin{abstract}
Introduction: This study aims to determine the effect of a twelve-month neuro-mobilization intervention added to standard physiotherapy (PT) method on the back pain compared to standard PT method alone in patients with adult idiopathic scoliosis and pain. Methodology: 36 patients with adolescent-onset adult thoracolumbar idiopathic scoliosis were randomized to the Experimental (EG) or Control group (CG). The standard PT method, which includes autocorrection in 3D, integration in daily life, stabilizing the corrected posture, and patient education consisted of a 30 minute daily home program and weekly supervised sessions by a physiotherapist. The neuro-mobilization exercise was performed from supine position with straight leg raised repetitive active ankle dorsiflexion. Patient outcomes included visual analogue scale (VAS) for pain, the angle of trunk rotation (ATR), and Cobb angles measured at baseline, and at 12-months follow-up. The collected data were statistically treated $(p \leq 0.05)$. Results: The statistical processing of the data shows a significant Cobb angles and ATR improvement in both groups of patients. In EG Cobb angle decreased from $34.7 \pm 3.9^{\circ}$ to $27.6 \pm 4.1^{\circ}$, ATR from $10.7 \pm 2.5^{\circ}$ to $5.6 \pm 1.3^{\circ}$, and pain score from $3.7 \pm 1.1$ to $0.6 \pm 0.9(p<0.05)$. A change of $5^{\circ} \mathrm{Cobb}$ angle was considered clinically significant. In CG Cobb angle decreased from $33.6 \pm 3.4^{\circ}$ to $30.6 \pm 4.7^{\circ}$, ATR from $10.6 \pm 2.2^{\circ}$ to $7.9 \pm 1.2^{\circ}$, and pain score from $3.8 \pm 1.3$ to $2.9 \pm 1.2(p<0.05)$. Discussion and conclusions: The comparative analysis of the final results between the two groups showed better pain outcomes in the EG patients $(p<0.05)$. These findings indicate a connection between the mobility of the spinal cord and the pain. Neuro-mobilization added to the standard PT method was superior compared to standard PT method alone for reducing the pain and curve severity in patients with adult idiopathic scoliosis.
\end{abstract}

Key words: scoliosis, neuromobilization, physiotherapy.

\section{INTRODUCTION}

The impact of scoliosis during adulthood is correlated with two main parameters: the frontal curve and the sagittal profile. The Cobb angle, measured in the frontal plane, is associated with the risk of progression of the deformity, this being negligible for curves below $30^{\circ} \mathrm{Cobb}$, and very high for curves over $50^{\circ} \mathrm{Cobb}$. The impairment of the sagittal profile is predictive of back pain and disability. For scoliosis patients with chronic back pain, the main approach according to current practice is the surgical one, but there are relevant risks and side effects. For these reasons many patients seek for a non-operative treatment.

There are very few papers concerning physiotherapy for adult scoliosis. In a systematic literature study (Everett, Patel, 2007) on the non-surgical treatment of scoliosis after completion of bone structure growth, the authors established only 10 articles in the Medline, PubMed and CINAHL associated with the use of physiotherapy in these patients. The relationship between pain and scoliosis has been studied (Weiss et al., 1998), it is noted that in adults, scoliosis is associated with spinal pain in $60-80 \%$ of patients, neurological symptoms (nerve root compression from the concave side with radial pain), imbalance in sagittal and coronal plan, which also leads to back pain (Glassman et al., 2005). Attention is drawn to the need of study and improvement of the quality of life in women with idiopathic scoliosis. A number of authors (Asher, Burton, 2006; Negrini et al., 2006, 2012, 2015; Weinstein et al., 2003; Zadeh, Gleiber, 2015) recommend a report of the results for a clinically significant period and number of patients at the start and end of treatment with a scoliosis angle above $10^{\circ}$, above $30^{\circ}$, and over $50^{\circ}$ Cobb. There is currently a lack of consensus on the most efficacious conservative treatments for these patients.

\section{Aim and objectives of the study}

This study aims to determine the effect of a twelvemonth neuro-mobilization intervention added to standard physiotherapy method (Experimental group) on the pain and deformity compared to standard physiotherapy method alone (Control group) in patients with adult idiopathic scoliosis and pain. 


\section{METHODS}

All adult idiopathic scoliosis patients attended our practice in the period 2006-2017 were analyzed.

The specific inclusion criteria were:

- Having a diagnosis with adolescent-onset adult idiopathic scoliosis

- Cobb angle between 30 and $40^{\circ}$

- Having the main deformity in the thoracolumbar region and chronic low back pain

- Without neurological symptoms

- Age between 20 and 35 years

- Risser 5

- $\geq 1$ years' follow-up.

Patients who had previous surgery to spine or limb, or still wearing a brace for scoliosis were excluded. A total of 36 patients with adolescent-onset adult thoracolumbar (TL) idiopathic scoliosis met the inclusion criteria and were randomized to the Experimental (EG - 18 patients) or Control group (CG 18 patients). The patients were aged between 22-35 years. No differences were found between groups in terms of demographic or radiographic pretreatment data. Age (EG: $27.8 \pm 5.07$ vs. CG: $28.2 \pm 6.5 \mathrm{yr}$; $\mathrm{a}=0.19$ ), coronal TL Cobb (EG: $34.72^{\circ} \pm 3.9$ vs. CG: $\left.33.5^{\circ} \pm 3.38 ; \mathrm{a}=0.94\right)$ were similar. The angle of curvature varied from $30^{\circ}$ to $40^{\circ}$ (mean $35.7 \pm 4.2^{\circ} \mathrm{Cobb}$ ) and Risser grade 5. No differences were found in pelvic parameters (pelvic incidence, pelvic tilt, sacral slope), apical translation, coronal balance, lumbar rotatory subluxation, or lumbar lordosis.

The group of patients was dominated by women $32 / 36(88.9 \%)$ and $4 / 36$ (11.1\%). The patients' characteristics were similar between groups.

The main goal of physiotherapy (PT) was to provide pain relief and improve functional lifestyle with minimum intervention.

We applied the "sagittal realignment test" (SRT) and patients reported significant pain release in the SRT (Weiss, Werkmann, 2009). We teach all patients in auto-SRT and Relordosation techniques (Weiss, Klein, 2006; Machado et al., 2006; Browder et al., 2007) to improve/correct the sagittal profile aiming at a balanced " $\mathrm{S}$ " when viewed from lateral.

The standard PT method, which includes auto-correction in $3 \mathrm{D}$, integration in daily life, stabilizing the corrected posture, and patient education consisted of a 30 minute daily home program and weekly supervised sessions by a physiotherapist.

The neuro-mobilization exercise was performed from supine position with straight leg raised repetitive active ankle dorsiflexion (20 repetitions, 2 times daily).

Patient outcomes included visual analogue scale (VAS) for pain, the angle of trunk rotation (ATR), and Cobb angles measured by radiological images at baseline, and at 12-months follow-up. The collected data were statistically treated by using SPSS software, version 18.0, and the following values were found: minimum, maximum, mean, S.D., and one sample paired t-test to compare between pre- and post-treatment in results in the group. The unpaired t-test was used to compare pre- and posttreatment results between two groups, at a confidence level of $\mathrm{p} \leq 0.05$.

\section{RESULTS}

The statistical processing of the data shows a significant Cobb angles and ATR improvement in both groups of patients. In EG Cobb angle decreased from $34.7 \pm 3.9^{\circ}$ to $27.6 \pm 4.1^{\circ}, \Delta \mathrm{X}=7.1, \mathrm{p}<0.05$ (Figure $3)$. A change of $5^{\circ}$ Cobb angle was considered clinically significant. In CG Cobb angle decreased from $33.6 \pm 3.4^{\circ}$ to $30.6 \pm 4.7^{\circ}, \Delta \mathrm{X}=2.9, \mathrm{p}<0.05$ (Table 1 ).

Table 1. Cobb angle - results after PT

\begin{tabular}{|l|l|l|l|l|l|l|}
\hline \multicolumn{1}{|c|}{ EG } & \multicolumn{1}{c|}{ CG } & & & & & \\
\hline average X1 & average X2 & $\Delta \mathbf{X}$ & $\mathbf{S 1}$ & $\mathbf{S 2}$ & $\mathbf{t}$ & $\boldsymbol{\alpha}$ \\
\hline 27.61 & 30.66 & -3.05 & 4.11 & 4.7 & 2.07 & 0.05 \\
\hline
\end{tabular}

At follow-up, the radiological deformities showed a In EG the angle of trunk rotation (ATR) decreased significant, although not clinically meaningful, bet- from $10.7 \pm 2.5^{\circ}$ to $5.6 \pm 1.3^{\circ}, \mathrm{p}<0.05$. In CG the ween-group difference of $3^{\circ} \mathrm{Cobb}$ angle in favor of ATR decreased from $10.6 \pm 2.2^{\circ}$ to $7.9 \pm 1.2^{\circ}, \mathrm{p}<0.05$. the EG.

The statistical processing of the data shows a significant improvement in both groups. Therefore, 
the applied PT in both groups led to significant improvement in the posture. The comparative analysis of outcomes between the two groups showed statistically significant better results in EG compared to
CG. At the end of the treatment, the ATR showed a significant, although not clinically meaningful, between-group difference of $2.33^{\circ}$ in favor of the EG (Table 2).

Table 2. ATR at the end of the treatment

\begin{tabular}{|l|l|l|l|l|l|l|}
\hline \multicolumn{1}{|c|}{ EG } & CG & & & & & \\
\hline average X1 & average X2 & $\Delta \mathbf{X}$ & $\mathbf{S 1}$ & \multicolumn{1}{c|}{ S2 } & $\mathbf{t}$ & \\
\hline 5.61 & 7.94 & -2.33 & 1.28 & 1.16 & 5.7 & 0.001 \\
\hline
\end{tabular}

The auto-correction in 3D, integration in daily life, stabilizing the corrected posture, and patient education has helped the patients to improve theirs posture, awareness of functional movement, and over all well-being. The integration of concepts of movement reeducation based on bio mechanics and motor development has helped the improvement of proprioception and balance, and to release the internal compression caused by gravity. Obvi- ously, also the increased quality of movement and the biomechanical changes of the spinal soft tissues could play a role in decreasing the risk of progression (Stokes, Burwell, Dangerfield, 2006; De Mauroy, 2008).

The variation analysis of VAS data for pain (Table 3) showed that the results in EG and CG were initially comparable.

Table 3. Variation analysis of VAS data for pain.

\begin{tabular}{|l|l|l|l|l|l|l|l|}
\hline & \multicolumn{1}{|c|}{ EG } & \multicolumn{1}{c|}{ CG } & & & & & \\
\hline & \multicolumn{1}{|c|}{ average X1 } & average X2 & \multicolumn{1}{c|}{$\Delta \mathbf{X} \mathbf{( X 1 - X 2 )}$} & $\mathbf{S 1}$ & \multicolumn{1}{c|}{ S2 } & $\mathbf{t}$ & $\boldsymbol{\alpha}$ \\
\hline Beginning & 3.67 & 3.78 & -0.11 & 1.13 & 1.26 & 0.27 & - \\
\hline End & 0.61 & 2.94 & -2.33 & 0.97 & 1.16 & 6.51 & 0,001 \\
\hline$\Delta \mathrm{X}$ & 3,06 & 0,84 & & & & & \\
\hline $\mathrm{t}$ & 8,64 & 2,06 & & & & & \\
\hline$\alpha$ & 0,001 & 0,05 & & & & & \\
\hline
\end{tabular}

The analysis of the final results between the two groups showed better pain outcomes in the EG patients compared to CG patients $(\mathrm{p}<0.05)$. In EG pain score decreased from $3.7 \pm 1.1$ to $0.6 \pm 0.9$, $\mathrm{p}<0.05$. In CG pain score decreased from $3.8 \pm 1.3$ to $2.9 \pm 1.2, \mathrm{p}<0.05$. We associated the more expressed positive change in EG patients with the use of neuro-mobilization exercise. These findings indicate a connection between the mobility of the spinal cord and the pain. Therefore, we propose to perform neuro-mobilization exercise in conjunction with scoliosis specific exercises in the treatment of patients with adult idiopathic scoliosis and chronic back pain. However, it should be pointed that all subjects in our study had a good flexibility, which may contribute to the outcome. After the treatment the patients were pain free, and living a functional and active life. Stability or improvement of more than $5^{\circ}$ in $80 \%$ may be an alternative to surgery. The addition of neuro-mobilization exercise with the integration of specific movement reeducation methods for scoliosis as well as auto-correction in $3 \mathrm{D}$, integration in daily life, and stabilizing the corrected posture can be very useful to help the well-being and function for adults with severe idiopathic scoliosis, slowing down the processes of deterioration also caused by aging.

\section{DISCUSSION}

There is scant literature about conservative treatments for adult patients with scoliosis so far. There is a much lower potential for progression of idiopathic scoliosis (IS) after the spinal growth is complete. In adulthood, IS may intensify as a result of progressive osseous deformities and collapsing of the spine (Negrini et al., 2012). This phenomenon is reported especially in scoliosis that is more severe than $50^{\circ}$, while the risk of progression starts to increase as the curve grows above $30^{\circ}$ (Willner, Uden, 1982; Negrini, et al., 2006) less severe IS curves often remain stable. Nevertheless, the natural history of adult scoliosis is not well known to date, and it is still possible the progression can have some peak periods (Negrini et al., 2008). Recently some pa- 
pers appeared reporting about bracing and exercises reporting the chance to effectively reduce pain, increase quality of life and prevent or stop progression. While in adolescents exercises' aim is to reduce postural collapse and rehabilitate movement in this way allowing a better growth of the vertebrae (Negrini, 2007; Stokes, Burwell, Dangerfield, 2006) in adults the process to be controlled is bony deformation due to degeneration. Again, postural collapse can have a role, in the long term, of facilitating progression because of chronic asymmetric increase of compression (Negrini et al., 2008). Treatment for adult scoliosis is generally based on symptoms and not the size of the curve. According to Pizones et al. (2017) function and clinical symptoms are the main factors that motivate thoracolumbar adult scoliosis patients to pursue surgery. Demographic and radiographic parameters did not seem to influence decision-making.

The functional outcomes gained by us in patients with adult scoliosis confirm researches by other authors who claim that application of specific physiotherapeutic exercise in scoliosis affects positively patients, in comparison with natural history of IS after 18 years of age (Negrini et al., 2015). Data obtained by us is comparable and better than those described by other authors (Morningstar, 2011, 2012). Scoliosis in adults is associated with lower quality of life (Shapiro, Taira, Boachie-Adjei, 2003) low results from health questionnaire SF-36 (Schwab et al., 2003) and prone to development of chronic pain, more often compared to the general population (Weinstein et al., 2003). In actual fact scoliosis does not automatically induce spinal or low back pain (Asher, Burton, 2006) and therefore the type of low back pain as reported in this study should not be restricted to the existence of a spinal deformity. A study with over 2000 scoliosis patients with pain reveals a lack of correlation of pain intensity and curve magnitude (Weiss et al., 1998).

\section{CONCLUSION}

The standard PT method improved deformity. Neuro-mobilization added to the standard PT method was superior compared to standard PT method alone for reducing the pain and curve severity in patients with adult idiopathic scoliosis.

The lack of consensual, effective, non-surgical scoliosis treatment of these patients, imposes the necessity of the continuation and expansion of research in this area, the establishment of evidence-based clinical guidelines on physiotherapy method, that effectively improves the functional capacities and the patients' quality of life.

\section{REFERENCES}

Asher M., Burton D. (2006). Adolescent idiopathic scoliosis: natural history and long term treatment effects, Scoliosis, 1(1):2. doi:10.1186/1748-7161-1-2.

Browder D., Childs J., Cleland J., Fritz J. (2007). Effectiveness of an extension-oriented treatment approach in a subgroup of subjects with low back pain: a randomized clinical trial. Phys Ther, 87(12), pp. 1608-18. De Mauroy J. (2008). Idiopathic scoliosis and chaos. Stud Health Technol Inform, 135, pp. 53-57.

Everett C., Patel R. (2007). A systematic literature review of nonsurgical treatment in adult scoliosis, Spine, Vol. 32 No.9, pp. 130-4. 1(1):2. doi:10.1186/1748-7161-1-2.

Glassman S., Bridwell K., Dimar J., Horton W., Berven S., Schwab F. (2005). The impact of positive sagittal balance in adult spinal deformity, Spine (Phila Pa 1976), Vol. 30 No.18, pp. 2024-9.

Machado L., de Souza M., Ferreira P., Ferreira M. (2006). The McKenzie method for low back pain: a systematic review of the literature with a meta-analysis approach. Spine 2006, 31(9):E, pp. 254-62.

Morningstar M. (2011). Outcomes For Adult Scoliosis Patients Receiving Chiropractic Rehabilitation: A 24-month Retrospective Analysis, J Chiropractic Medicine, Vol. 10 No.3, pp. 179-184.

Morningstar M. (2012). Scoliosis and ARC3D Therapy: Restoring Hope With Non-Surgical Treatment, Infinity Publishing, pp. 86-96.

Negrini A., Parzini S., Negrini M., Romano M., Atanasio S., Zaina F., Negrini S. (2008). Adult scoliosis can be reduced through specific SEAS exercises: a case report. Scoliosis, 3:20.

Negrini A., Negrini M., Donzelli S., Romano M., Zaina F., Negrini S. (2015). Scoliosis-Specific exercises can reduce the progression of severe curves in adult idiopathic scoliosis: a long-term cohort study, Scoliosis, 10:20. 10.1186/s13013-015-0044-9.

Negrini S., Grivas T., Kotwicki T., Maruyama T., Rigo M., Weiss H.. (2006). Members of the Scientific society On Scoliosis Orthopaedic and Rehabilitation Treatment (SOSORT): why do we treat adolescent idiopathic scoliosis? What we want to obtain and to avoid for our patients: SOSORT 2005 consensus paper, Scoliosis, 1:4. doi: 10.1186/1748-7161-1-4.

Negrini S. (2007). The Evidence-Based ISICO Approach to Spinal Deformities. 1st edition. Milan, Boston: ISICO. Negrini S., Aulisa A., Aulisa L., Circo A., De Mauroy 
J., Durmala J. et al. (2012). 2011 SOSORT guidelines: orthopaedic and rehabilitation treatment of idiopathic scoliosis during growth, Scoliosis, 7(1):3. doi: 10.1186/1748-7161-7-3.

Pizones J., Pérez Martin-Buitrago M., Perez-Grueso F., Vila-Casademunt A., Alanay A., Obeid I., Kleinstück F., Acaroglu E., Pellisé F. (2017). Function and Clinical Symptoms are the Main Factors that Motivate Thoracolumbar Adult Scoliosis Patients to Pursue Surgery. Spine (Phila Pa 1976), Jan 1;42(1):E31-E36.

Schwab F., Dubey A., Pagala M., Gamez L., Farcy J. (2003). Adult scoliosis: a health assessment analysis by SF-36, Spine, Vol. 28 No.6, pp. 602-606.

Shapiro G., Taira G., Boachie-Adjei O. (2003). Results of surgical treatment of adult idiopathic scoliosis with low back pain and spinal stenosis: a study of long-term clinical radiographic outcomes, Spine, Vol. 28 No.6, pp. 358-363.

Stokes I., Burwell R., Dangerfield P. (2006). Biomechanical spinal growth modulation and progressive adolescent scoliosis - a test of the 'vicious cycle' pathogenetic hypothesis: Summary of an electronic focus group debate of the IBSE. Scoliosis, 1:16.

Weinstein S., Dolan L., Spratt K., Peterson K., Spoonamore M., Ponseti I.. (2003). Health and function of patients with untreated idiopathic scoliosis: a 50-year natural history study, JAMA, 289(5):559-67. doi:10.1001/ jama.289.5.559.

Weiss H., Verres C., Lohschmidt K., El Obeidi N. (1998).

Pain and scoliosis - is there any relationship?, Orthop Prax, Vol. 34, pp. 602-606.

Weiss H., Klein R. (2006). Improving excellence in scoliosis rehabilitation: a controlled study of matched pairs. Pediatr Rehabil, 9(3), pp. 190-200.

Weiss, H., Werkmann, M. (2009). Unspecific chronic low back pain - a simple functional classification tested in a case series of patients with spinal deformities. Scoliosis, 4:4 doi:10.1186/1748-7161-4-4.

Willner S., Uden A. (1982). A prospective prevalence study of scoliosis in Southern Sweden. Acta Orthop Scand, 53(2), pp. 233-237.

Zadeh J., Gleiber M. (2015):. Adolescent Idiopathic Scoliosis: An in Depth Analysis and Historical Review, MOJ Orthop Rheumatol, Vol. 3 No.4, pp. 306-34.

Corresponding author: Prof. Evgeniya Dimitrova, DSc National Sports Academy „Vassil Levski“ Sofia, Bulgaria E-mail: janydim@abv.bg 